\title{
ON PLANAR CREMONA MAPS OF PRIME ORDER
}

\author{
TOMMASO DE FERNEX
}

\begin{abstract}
This paper contains a new proof of the classification of prime order elements of $\operatorname{Bir}\left(\mathbb{P}^{2}\right)$ up to conjugation. The first results on this topic can be traced back to classic works by Bertini and Kantor, among others. The innovation introduced by this paper consists of explicit geometric constructions of these Cremona transformations and the parameterization of their conjugacy classes. The methods employed here are inspired to [4], and rely on the reduction of the problem to classifying prime order automorphisms of rational surfaces. This classification is completed by combining equivariant Mori theory to the analysis of the action on anticanonical rings, which leads to characterize the cases that occur by explicit equations (see [28] for a different approach). Analogous constructions in higher dimensions are also discussed.
\end{abstract}

\section{Introduction}

One of the first contributions to the classification of conjugacy classes in the Cremona group of $\mathbb{P}^{2}$ can be attributed to Bertini for his work on birational involutions [6]. The classification of all finite subgroups of $\operatorname{Bir}\left(\mathbb{P}^{2}\right)$ up to conjugation was successively completed by Kantor in [19]. On the same topic, one also finds [27], [2], [15] and [12]. The classification of finite order planar Cremona maps, up to conjugation, is equivalent to the classification of normal multiple rational planes, up to birational equivalence. In this area one can find the results of Bottari [7] and Castelnuovo and Enriques [10]. Recently, Bayle and Beauville [4] and Calabri [8], [9] gave new proofs of the birational classification of, respectively, involutions in $\operatorname{Bir}\left(\mathbb{P}^{2}\right)$ and double and triple rational planes. Closely related to these topics are results leading towards the determination of automorphism groups of rational surfaces, such as [26], [23], [22], [14], [20], [17], [18] [28] and [29]. We refer to [1] for an account of the classic theory of planar Cremona maps.

The purpose of this paper is to give a new proof of the birational classification of planar Cremona maps of prime order, aiming a better under-

Received July 31, 2002.

Revised January 9, 2003, March 25, 2003.

2000 Mathematics Subject Classification: Primary 14E07, 14J50; secondary 14E20. 
standing of the geometry governing these transformations. Elements representing each conjugacy class will be constructed by first realizing them as automorphisms on birational equivalent models, and then interpreting the constructions in terms of the geometry of $\mathbb{P}^{2}$. Parameterization of their classes will follow from this approach.

Extending the methods in [4], the classification of Cremona maps of prime order is reduced, through a suitable resolution of indeterminacy, to that of automorphisms of prime order of smooth rational surfaces. In fact, we will deal with automorphisms of prime order of smooth projective surfaces whose canonical class is not numerically effective. The classification splits into two categories, according to the rank of the invariant part of the Nerón-Severi group of the surface. If this rank is at least 2, we apply Mori theory in the spirit of [4] and [28], searching for equivariant fibration structures. Otherwise the rank is 1 . Then, after observing that the surface is Del Pezzo, the classification is completed by considering the action that the automorphism induces on the anticanonical ring. This approach enables us to characterize each case by explicit equations and identify families of analogous automorphisms in all dimensions.

The classification of automorphisms of prime order of smooth rational surfaces has been already proved, by different methods, by Dolgachev and Zhang in their very nice paper [28]. We would like to mention that Theorem A below differs from [28, Theorem 1] in the way certain cases are characterized: in [28] surfaces and automorphisms are constructed and characterized as cyclic coverings over their quotients, whereas in this paper we characterize them in terms of their equations.

This paper is organized as follows. The main results of classification, given in Theorems A, B, E, F, are stated in Section 1. Sections 2 and 3 are respectively devoted to fix the notation and present some preliminary material. Section 4 contains the proof of Theorems A and B. In Sections 5 and 6, we see two more properties concerning automorphisms of surfaces: Propositions C and D. Finally, in Section 6, Theorems A, B and Propositions C, D are applied to prove Theorems E and F. Special numeration, labeling certain cases, will be consistently adopted in all statements.

Acknowledgements. First and foremost, I would like to express my gratitude to Professor L. Ein for his invaluable advice and help throughout the preparation of this paper. It is a pleasure to thank A. Beauville, N. Budur, A. Lanteri and R. Lazarsfeld for several useful discussions, and 
I. Dolgachev and D.-Q. Zhang for precious comments and for sending me their preprint while I was working on this problem. I would like to thank the referee for his corrections and for indicating an alternative argument that simplifies the original proofs of Proposition 4.3.1 and Lemma 4.4.1. I am grateful to A. Albano, M. Alberich, A. Calabri, L. Caporaso, L. Chiantini, F. Russo, and F.L. Zak for very interesting comments. I would like to thank the University of Genova, the University of Hong Kong and the University of Illinois at Chicago for their hospitality, and the MURST of the Italian Government for partial support in the framework of the National Research Project (Cofin 2000) "Geometry on Algebraic Varieties".

\section{$\S 1$. The main results}

\subsection{Automorphisms of prime order of surfaces}

We work over the field of complex numbers. Let $X$ be a smooth projective surface, and $\sigma \in \operatorname{Aut}(X)$. The pair $(X, \sigma)$ is said to be minimal if for any birational morphism $\phi: X \rightarrow X^{\prime}$ such that $X^{\prime}$ is smooth and $\phi \sigma \phi^{-1} \in \operatorname{Aut}\left(X^{\prime}\right), \phi$ is an isomorphism. Examples of minimal pairs are given by the following two classic involutions, whose constructions we recall here for the convenience of the reader. If $X$ is a smooth Del Pezzo surface of degree 2, the linear system $\left|-K_{X}\right|$ defines a double covering over $\mathbb{P}^{2}$, branched along a smooth quartic curve; the involution defined by this cover is called Geiser involution. Similarly, if $X$ is a smooth Del Pezzo surface of degree 1 , the linear system $\left|-2 K_{X}\right|$ defines a double covering over a quadric cone, branched along the vertex of the cone and a smooth curve of genus 4, and the corresponding involution is the Bertini involution of $X$.

THEOREM A. Let $X$ be a smooth projective surface whose canonical class is not nef, and $\sigma \in \operatorname{Aut}(X)$ be an element of prime order $n$ such that the pair $(X, \sigma)$ is minimal. Then either $(X, \sigma)$ is one of the following (where any value of $n$ may occur) :

1. $X \cong \mathbb{P}^{2}$ and $\sigma \in \operatorname{PGL}(3)$;

2. $X$ is a geometrically ruled surface and $\sigma$ is fiberwise, either inducing an effective automorphism on the base curve of the ruling or restricting to an effective automorphism on each fiber;

or $n=2$ and $(X, \sigma)$ is one of the following:

3. $X$ is a conic bundle and $\sigma$ restricts to an effective involution on each fiber; the two components of each singular fiber are flipped by $\sigma$; 
4. $X \cong \mathbb{P}^{1} \times \mathbb{P}^{1}$ and $\sigma$ is the involution swapping the two rulings of $X$;

5. $X$ is a Del Pezzo surface of degree 2 and $\sigma$ is the Geiser involution;

6. $X$ is a Del Pezzo surface of degree 1 and $\sigma$ is the Bertini involution; or $n=3$ and $(X, \sigma)$ is one of the following:

A1. $X$ is a Del Pezzo cubic surface defined by an equation of the form $x^{3}=F(y, z, w)$ in $\mathbb{P}^{3}$, and $\sigma$ is the restriction of the automorphism of $\mathbb{P}^{3}$ given by $(x, y, z, w) \rightarrow(\lambda x, y, z, w)$, where $\lambda \neq 1$ is a 3rd-root of unity;

A2. $X$ is a Del Pezzo sextic surface defined by an equation of the form $z^{3}=$ $F(x, y, w)$ in the weighted projective space $\mathbb{P}(1,1,2,3)$ with coordinates $(x, y, z, w)$, and $\sigma$ is the restriction of the automorphism of $\mathbb{P}(1,1,2,3)$ given by $(x, y, z, w) \rightarrow(x, y, \lambda z, w)$, where $\lambda \neq 1$ is a 3rd-root of unity;

or $n=5$ and $(X, \sigma)$ is one of the following:

A3. $X$ is a Del Pezzo sextic surface defined by an equation of the form $x y^{5}=F(x, z, w)$ in the weighted projective space $\mathbb{P}(1,1,2,3)$ with coordinates $(x, y, z, w)$, and $\sigma$ is the restriction of the automorphism of $\mathbb{P}(1,1,2,3)$ given by $(x, y, z, w) \rightarrow(x, \lambda y, z, w)$, where $\lambda \neq 1$ is a 5th-root of unity;

A4. $X$ is the Del Pezzo surface $\mathrm{Bl}_{\Sigma} \mathbb{P}^{2}$, where $\Sigma$ is the set of four points in general position, and $\sigma$ is the lift over $X$ of the birational transformation of $\mathbb{P}^{2}$ given, for suitable coordinates of $\mathbb{P}^{2}$, by $(x, y, z) \rightarrow$ $(x(z-y), z(x-y), x z)$.

Moreover, a smooth sextic surface $X$ in $\mathbb{P}(1,1,2,3)$ admits both automorphisms $\sigma_{2}, \sigma_{3}$ such that, for $i=2,3$, the pair $\left(X, \sigma_{i}\right)$ is as in case Ai if and only if, in suitable coordinates $(x, y, z, w), X$ is defined by $x^{6}+x y^{5}+z^{3}+$ $w^{2}=0$.

Notation 1.1.1. We will denote by $X_{0}$ the sextic surface in $\mathbb{P}(1,1,2,3)$ defined by the equation $x^{6}+x y^{5}+z^{3}+w^{2}=0$.

Remark 1.1.2. Even if we just assumed that the canonical class is not nef, all surfaces appearing in the classification have Kodaira dimension $-\infty$. This is expected, since $\operatorname{Bir}(Y)=\operatorname{Aut}(Y)$ for any smooth surface $Y$ with nef canonical class.

Theorem B. Let $(X, \sigma)$ be as in one of cases A1-A4 of Theorem A. Denote by $f: X \rightarrow X / \sigma$ the quotient map. 
B1. If $(X, \sigma)$ is as in case $\mathrm{A} 1$, then $X$ is a "special" Del Pezzo surface of degree 3 , and $X / \sigma \cong \mathbb{P}^{2}$. Moreover, $f$ is defined by the 2-dimensional linear subsystem of $\left|-K_{X}\right|$ spanned by the orbits of $(-1)$-curves of $X$ (see Definition 4.1.3), and is totally ramified over a smooth plane cubic curve.

B2. If $(X, \sigma)$ is as in case A2, then $X$ is a "special" Del Pezzo surface of degree 1 , and $X / \sigma \cong \overline{\mathbb{F}}_{3}$, the cone in $\mathbb{P}^{4}$ over a rational twisted cubic. Moreover, $f$ is defined by the linear subsystem of $\left|-3 K_{X}\right|$ spanned by $3 C_{x}, 2 C_{x}+C_{y}, C_{x}+2 C_{y}, 3 C_{y}, C_{w}$ (see Notation 3.2.1), and is totally ramified over the vertex of the cone and the three-canonical model of a smooth curve of genus 2 .

B3. If $(X, \sigma)$ is as in case $\mathrm{A} 3$, then $X$ is a "special" Del Pezzo surface of degree 1 , and $X \cong X_{0}\left(X_{0}\right.$ is defined in Notation 1.1.1 above $)$ if and only if $j(C)=0$ for some (equivalently, for every) smooth $C \in\left|-K_{X}\right|$. In all cases, $X / \sigma$ is isomorphic to the sextic hypersurface of equation $x u=F(x, z, w)$ in the weighted projective space $\mathbb{P}(1,2,3,5)$ with coordinates $(x, z, w, u) ; X / \sigma$ can be realized by contracting the curve $G^{\prime} \cup S_{0}$ of $Z_{22}$ if $X \cong X_{0}$, and of $Z_{211}$ otherwise (see Notation 2.0.6). Moreover, $f$ is defined by the linear subsystem of $\left|-3 K_{X}\right|$ spanned by $5 C_{x}, 5 C_{y}, 3 C_{x}+C_{z}, 2 C_{x}+C_{w}, C_{z}+C_{w}$ (see Notation 3.2.1), and is totally ramified over the singular point of $X / \sigma$ and a smooth elliptic curve.

B4. If $(X, \sigma)$ is as in case A4, then $X$ is the Del Pezzo surface of degree 5, and $X / \sigma$ is the image of $Z_{5511}$ under the contraction of $G_{1}^{\prime} \cup G_{2}^{\prime} \cup S_{0}$ (see Notation 2.0.6). Moreover, $f$ is totally ramified over the two singular points of $X / \sigma$.

Remark 1.1.3. The "specialty" mentioned in cases B1-B3 is characterized by the constraints given to the equation defining $X$ (see A1-A3). It is known that, apart of the Bertini involution, there are no other automorphisms on general Del Pezzo surfaces of degree 1 or 3 (see [20]). The information on the linear systems defining $f$, given for cases B1-B3, will be used to describe the birational transformations they induce on $\mathbb{P}^{2}$. This is not needed for case B4 (one can show that, in this case, $f$ is defined a linear subsystem of $\left.\left|-3 K_{X}\right|\right)$.

\subsection{Analogous constructions in higher dimensions}

Del Pezzo manifolds of dimension $N \geq 3$ are classified by Fujita [13]. Del Pezzo manifolds of degree 3 admit projective embeddings as cubic hy- 
persurfaces, and we find in those which are defined by an equation of the form $x_{0}^{3}=F\left(x_{1}, \ldots, x_{N+1}\right)$ the analogues of case A1.

If $X$ is a Del Pezzo manifold of degree 2, then $X$ is a quartic hypersurface in the weighted projective space $\mathbb{P}\left(1^{N+1}, 2\right)$, and the linear projection $\mathbb{P}\left(1^{N+1}, 2\right) \rightarrow \mathbb{P}\left(1^{N+1}\right)$ induces a double covering of $X$ over $\mathbb{P}^{N}$. This construction generalizes the Geiser involution.

Let now $X$ be a Del Pezzo manifold of degree $1 . \quad X$ is a sextic hypersurface in $\mathbb{P}\left(1^{N}, 2,3\right)$. The linear projection $\mathbb{P}\left(1^{N}, 2,3\right) \rightarrow \mathbb{P}\left(1^{N}, 2\right)$ induces a double covering of $X$ over the cone over Veronese variety $v_{2}\left(\mathbb{P}^{N}\right)$. This is the higher dimensional analogue of the Bertini involution. If we additionally assume that $X$ is defined, in suitable weighted coordinates $\left(x_{1}, \ldots, x_{N}, z, w\right)$, by an equation of the form $z^{3}=F\left(x_{1}, \ldots, x_{N}, w\right)$, then the linear projection $\mathbb{P}\left(1^{N}, 2,3\right) \rightarrow \mathbb{P}\left(1^{N}, 3\right)$ induces a triple cyclic covering of $X$ over the cone over $v_{3}\left(\mathbb{P}^{N}\right)$. This extends case A2. Similarly, we can assume that $X$ is defined by an equation of the form $x_{2} x_{1}^{5}=$ $F\left(x_{2}, \ldots, x_{N}, z, w\right)$. Consider the action of order 5 defined on the ring $\mathbb{C}\left[x_{1}, \ldots, x_{N}, z, w\right]$ by sending $x_{1} \rightarrow \lambda x_{1}$, where $\lambda$ is a 5th-root of unity. Then the equation of $X$ is invariant with respect to this action, and the inclusion of the invariant subring of $\mathbb{C}\left[x_{1}, \ldots, x_{N}, z, w\right] /\left(x_{2} x_{1}^{5}-F\right)$ determines a degree 5 cyclic covering of $X$ over the sextic hypersurface of equation $x_{2} u=F\left(x_{2}, \ldots, x_{N}, z, w\right)$ in the weighted projective space $\mathbb{P}\left(1^{N-1}, 2,3,5\right)$ of coordinates $\left(x_{2}, \ldots, x_{N}, z, w, u\right)$. This extends case A3.

The remaining case to generalize is A4. The only Del Pezzo manifolds of degree 5 are the linear sections of the Grassmannian variety $\operatorname{Gr}(2,5)$ parametrizing lines in $\mathbb{P}^{4}$. The automorphism $\sigma$ of $X$ defined as in A4 extends to the higher dimensional Del Pezzo manifolds in the following way. Let $\sigma_{1}$ be a linear automorphism of order 5 of $\mathbb{C}^{5}$ admitting distinct eigenvalues $\lambda_{j}=e^{j 2 \pi i / 5}(j=0, \ldots, 4)$. Let $e_{0}, \ldots, e_{4} \in \mathbb{C}^{5}$ be the corresponding eigenvectors, and consider the basis $\left\{e_{i} \wedge e_{j} \mid 0 \leq i<j \leq 4\right\}$ for $\mathbb{C}^{5} \wedge \mathbb{C}^{5}$, hence the corresponding Plücker coordinates $p_{i j}$ of the projective space $\mathbb{P}^{9}$ in which $\operatorname{Gr}(2,5)$ is embedded. The automorphism $\sigma_{1}$ induces on $\mathbb{P}^{9}$ the automorphism $\sigma_{2}$, which sends $p_{i j} \rightarrow \lambda_{i} \lambda_{j} p_{i j}$, and the latter restricts to an automorphism $\sigma_{3}$ of $\operatorname{Gr}(2,5)$. Consider the five dimensional linear subspace $P \subset \mathbb{P}^{9}$ given by $p_{01}-p_{24}=p_{02}-p_{34}=p_{03}-p_{12}=p_{04}-p_{13}=0$. Then $X=P \cap \operatorname{Gr}(2,5)$ is a smooth Del Pezzo surface of degree $5, \sigma_{3}$ restricts to an automorphism $\sigma$ of $X$, and $(X, \sigma)$ is as in case A4. In a similar fashion, $\sigma_{3}$ restricts to automorphisms on the invariant Del Pezzo manifolds of intermediate dimensions. 


\subsection{Birational transformations of prime order of $\mathbb{P}^{2}$}

We first recall the definition of three celebrated birational involutions (Examples 1.3.1-1.3.3) and describe the constructions of four other birational transformations of $\mathbb{P}^{2}$ (Examples E1-E4). We would like to point out that, in these examples, not obvious facts will be claimed. Justifications of such claims are implicitly contained in the proof of Theorem E below.

EXAmPle 1.3.1. Let $C$ be a curve of degree $d \geq 3$ with an ordinary multiple point $q$ of multiplicity $d-2$. The de Jonquières involution of degree $d$ maps a general point $p \in \mathbb{P}^{2}$ to its harmonic conjugate on the line $L$ spanned by $p$ and $q$ with respect to the two residual points of intersection $q^{\prime}, q^{\prime \prime}$ of $L$ with $C$.

EXAMPLe 1.3.2. Let $\Sigma \subset \mathbb{P}^{2}$ be a set of 7 points in general position. The Geiser involution maps a general point $p \in \mathbb{P}^{2}$ to the ninth base point of the pencil of cubic $\left|\mathcal{O}_{\mathbb{P}^{2}}(3) \otimes I_{\Sigma} \otimes I_{p}\right|$.

EXAMPLE 1.3.3. Let $\Sigma \subset \mathbb{P}^{2}$ be a set of 8 points in general position. The Bertini involution maps a general point $p \in \mathbb{P}^{2}$ to the additional base point of the net of sextics $\left|\mathcal{O}_{\mathbb{P}^{2}}(6) \otimes I_{\Sigma}^{2} \otimes I_{p}\right|$.

ExAmple E1. Let $\Sigma \subset \mathbb{P}^{2}$ be a set of 6 points $p_{\alpha}$ such that $X=$ $\mathrm{Bl}_{\Sigma} \mathbb{P}^{2}$ is as in A1. Let $\gamma_{1}, \ldots, \gamma_{6}$ be the 6 conics passing through all but one point of $\Sigma$, and $\mathcal{L}$ denote the set of 15 lines passing through two of the six points of $\Sigma$. Then $\mathcal{L}$ splits in the union of subsets $\mathcal{L}=\mathcal{L}^{\prime} \cup \mathcal{L}_{1}^{\prime \prime} \cup \mathcal{L}_{2}^{\prime \prime} \cup \mathcal{L}_{3}^{\prime \prime}$, where $\mathcal{L}^{\prime}=\left\{L_{1}, \ldots, L_{6}\right\}$ and $\mathcal{L}_{\beta}^{\prime \prime}=\left\{L_{\beta, 1}, L_{\beta, 2}, L_{\beta, 3}\right\}(\beta=1,2,3)$, such that $L_{\alpha}$ is tangent to $\gamma_{\alpha}$ at $p_{\alpha}$ (for $\left.\alpha=1, \ldots, 6\right)$ and the three lines $L_{\beta, 1}, L_{\beta, 2}$ and $L_{\beta, 3}$ meet in one point (for $\beta=1,2,3$ ). The set of cubics of the form $D_{\alpha}=\gamma_{\alpha}+L_{\alpha}$ and $D_{\beta}=L_{\beta, 1}+L_{\beta, 2}+L_{\beta, 3}$ spans a net $W \subset\left|\mathcal{O}_{\mathbb{P}^{2}}(3) \otimes I_{\Sigma}\right|$. Imposing any extra general base point to $W$ gives two additional base points to the system, and permutations of such three points define two elements of order 3 of $\operatorname{Bir}\left(\mathbb{P}^{2}\right)$.

EXAMPle E2. Let $\Sigma \subset \mathbb{P}^{2}$ be a set of 8 points such that $X=\mathrm{Bl}_{\Sigma} \mathbb{P}^{2}$ is as in A2. Let $\Gamma_{w} \in\left|\mathcal{O}_{\mathbb{P}^{2}}(9) \otimes I_{\Sigma}^{3}\right|$ be a curve not contained in the span of the image of the triple embedding of $\left|\mathcal{O}_{\mathbb{P}^{2}}(3) \otimes I_{\Sigma}\right|$ in $\left|\mathcal{O}_{\mathbb{P}^{2}}(9) \otimes I_{\Sigma}^{3}\right|$, and let $W$ be the linear subsystem of $\left|\mathcal{O}_{\mathbb{P}^{2}}(9) \otimes I_{\Sigma}^{3}\right|$ spanned by the image of $\left|\mathcal{O}_{\mathbb{P}^{2}}(3) \otimes I_{\Sigma}\right|$ and $\Gamma_{w}$. For a suitable choice of $\Gamma_{w}$, any extra general base point imposed to $W$ carries with it two additional base points, and permutations of such three points define two elements of order 3 of $\operatorname{Bir}\left(\mathbb{P}^{2}\right)$. 
ExAmple E3. Let $\Sigma \subset \mathbb{P}^{2}$ be a set of 8 points such that $X=\mathrm{Bl}_{\Sigma} \mathbb{P}^{2}$ is as in A3. There are curves $\Gamma_{x}, \Gamma_{y}, \Gamma_{z}, \Gamma_{w}$ in $\left|\mathcal{O}_{\mathbb{P}^{2}}(3 a) \otimes I_{\Sigma}^{a}\right|$ (with $a=$ $1,1,2,3$, respectively) such that, if $W$ is the linear subsystem of $\mid \mathcal{O}_{\mathbb{P}^{2}}(15) \otimes$ $I_{\Sigma}^{5} \mid$ spanned by $5 \Gamma_{x}, 5 \Gamma_{y}, 3 \Gamma_{x}+\Gamma_{z}, 2 \Gamma_{x}+\Gamma_{w}, \Gamma_{z}+\Gamma_{w}$, then for any extra general base point imposed to $W$ the base locus incorporates four additional base points, and permutations of such five points define four elements of order 5 of $\operatorname{Bir}\left(\mathbb{P}^{2}\right)$.

ExAmple E4. In coordinates $(x, y, z)$ of $\mathbb{P}^{2}$, let $\tau:(x, y, z) \rightarrow(x(z-$ $y), z(x-y), x z)$. Then $\tau$ is an element of order 5 of $\operatorname{Bir}\left(\mathbb{P}^{2}\right)$.

TheOREM E. Examples E1-E4 above do define birational transformations of $\mathbb{P}^{2}$. Any element of prime order of $\operatorname{Bir}\left(\mathbb{P}^{2}\right)$ is conjugate to one and only one of the birational transformations described in Examples 1.3.1$\mathrm{E} 4$, or to an element of $\operatorname{Aut}\left(\mathbb{P}^{2}\right)$. Moreover, the transformations defined in these examples, with the possible exception of Example E4, are not conjugate to elements of $\operatorname{Aut}\left(\mathbb{P}^{2}\right)$.

Remark 1.3.4. It would be interesting to determine whether Example E4 is conjugate to an automorphism of $\mathbb{P}^{2}$. $^{1}$

The following theorem gives a description of the moduli spaces of conjugacy classes of prime order cyclic subgroups of $\operatorname{Bir}\left(\mathbb{P}^{2}\right)$. Let $\tau \in \operatorname{Bir}\left(\mathbb{P}^{2}\right)$ be an element and $G_{\tau} \subset \operatorname{Bir}\left(\mathbb{P}^{2}\right)$ be the cyclic subgroup generated by $\tau$. As in [4], associated to any such $\tau$ we consider the normalized fixed curve $\operatorname{NFC}(\tau)$. This is defined as the isomorphism class of the union of the irrational components of the normalization of the curve fixed by $\tau$. Since this is a birational invariant and is the same for every generator of the group $G_{\tau}$, we can define the correspondence

$$
\mathrm{NFC}:\left[G_{\tau}\right] \rightarrow \operatorname{NFC}(\tau)
$$

that associates to any conjugacy class $\left[G_{\tau}\right]$ of a cyclic subgroup of $\operatorname{Bir}\left(\mathbb{P}^{2}\right)$ the normalized fixed curve $\mathrm{NFC}(\tau)$ of any generator $\tau$ of any representative $G_{\tau}$ of the class.

TheOREM F. The map NFC naturally establishes one-to-one correspondences between:

\footnotetext{
${ }^{1}$ Recently, in [5], Beauville shows that the transformation defined in Example E4 is conjugated to an automorphism of the plane. In fact, it was brought to our attention by Dolgachev that this was already well known to Iskovskikh.
} 
1. conjugacy classes $\left[G_{\tau}\right]$, where $\tau$ is a de Jonquières involution of $\mathbb{P}^{2}$ of degree $d \geq 3$, and isomorphism classes of (hyper-)elliptic curves of genus $d-2 \geq 1$;

2. conjugacy classes $\left[G_{\tau}\right]$, where $\tau$ is a Geiser involution of $\mathbb{P}^{2}$, and isomorphism classes of non-hyperelliptic curves of genus 3 ;

3. conjugacy classes $\left[G_{\tau}\right]$, where $\tau$ is a Bertini involution of $\mathbb{P}^{2}$, and isomorphism classes of non-hyperelliptic curves of genus 4 whose canonical model lies on a singular quadric;

F1. conjugacy classes $\left[G_{\tau}\right]$, where $\tau$ is as in Example E1, and isomorphism classes of elliptic curves;

F2. conjugacy classes $\left[G_{\tau}\right]$, where $\tau$ is as in Example E2, and isomorphism classes of smooth curves of genus 2 ;

F3. conjugacy classes $\left[G_{\tau}\right]$, where $\tau$ is as in Example E3, and isomorphism classes of elliptic curves.

Remark 1.3.5. The four birational transforms $\tau, \tau^{2}, \tau^{3}, \tau^{4}$, where $\tau$ is as in Example E4, form a single conjugacy class (see Remark 4.6 .2 below). As pointed out to us by A. Beauville, linear automorphisms of order $n$, for any given $n<\infty$, form a single conjugacy class.

Proofs of Theorems E and F are contained in the last section.

\section{§2. Notation and conventions}

We work over an algebraically closed field $k$ of characteristic zero. We use standard notation accordingly to [16], [3] and [21]. We will adopt analogous notation as in [24] to denote certain elliptic fibrations over $\mathbb{P}^{1}$. In particular,

Notation 2.0.6. $\quad Z_{22}$ is the (unique) elliptic fibration having one section $S_{0}$, one singular fiber of type $I I$, and one of type $I I^{*} . Z_{211}$ is the (unique) elliptic fibration having one section $S_{0}$, two singular fibers of type $I_{1}$, and one of type $I I^{*} . Z_{5511}$ is the (unique) elliptic fibration having five (disjoint) sections $S_{0}, \ldots, S_{4}$, two singular fibers of type $I_{5}$, and two of type $I_{1}$. $Z_{5511}$ is obtained by resolving the base locus of the plane cubic pencil of equation $y(x-y)(x-z)+\lambda x z(y-z)=0$. We fix the following special notation: if $F_{0}$ is a fiber of type $I I^{*}$, then we write $F_{0}=G \cup G^{\prime}$ 
(set-theoretically), where $G$ is the irreducible component occurring with multiplicity 5 , and $G^{\prime}$ is the residual curve; if $F_{i}$ is a fiber of type $I_{5}$ of $Z_{5511}$, then we denote with $G_{i}^{\prime}$ the union of the four components of $F_{i}$ which are disjoint from $S_{0}(i=1,2)$.

Remark 2.0.7. To keep this paper more self-contained, we opted not to rely on the classification of extremal rational elliptic fibrations, which is given in [24]. We will only use the fact that the elliptic fibrations $Z_{22}, Z_{211}$ and $Z_{5511}$ are characterized by the data given in Notation 2.0.6

\section{$\S 3$. On the action of finite groups}

\subsection{Cyclic coverings}

Let $f: X \rightarrow Y$ be a cyclic cover, with $X$ a smooth projective variety of dimension $N$. Let $R \subset X$ be the set of points fixed by the Galois action, and set $B=f(R)$. Let $R=\sum R_{k}$ and $B=\sum B_{k}$ be the decompositions of these cycles with respect to the dimension of their components, the bottom index standing for the dimension. Actions of finite groups on smooth varieties can be locally linearized, up to passing to completion (see [3], page 85). This fact implies that $R$ is a smooth cycle and $\operatorname{Sing} Y \subseteq\left(B_{N-2} \cup \cdots \cup B_{0}\right)$. In particular, $Y$ is smooth if $N=1$, and $Y$ has only isolated singularities (contained in $B_{0}$ ) if $N=2$.

\subsection{Automorphisms on a Del Pezzo surface of degree 1}

If $X$ is a smooth Del Pezzo surface of degree 1, its anticanonical ring gives an embedding of $X$ into a weighted projective space $\mathbb{P}=\mathbb{P}(1,1,2,3)$. We identify $X$ with its image into this space. We can write $\mathbb{P}=\operatorname{Proj} S$, where $S=\mathbb{C}[x, y, z, w]$ is the ring graded by the conditions $\operatorname{deg} x=\operatorname{deg} y=$ $1, \operatorname{deg} z=2$ and $\operatorname{deg} w=3$.

Let $\sigma$ be an automorphism of $X$ of finite order $n$. Note that $\sigma$ lifts to an automorphism of the sheaf of differentials $\Omega_{X}$ of $X$. We deduce that $\sigma$ is extends to an automorphism of $\mathbb{P}$. In fact, we can find an automorphism $\sigma^{\prime}$ of $S$, of order $n$, that induces such automorphism of $\mathbb{P}$. One should observe that the choice of $\sigma^{\prime}$ is not unique.

Notation 3.2.1. We can assume that the coordinates $(x, y, z, w)$, chosen for $\mathbb{P}$, are equivariant, that is, that they define invariant divisors on $\mathbb{P}$. (Existence of such coordinates follows from the fact that linear automorphisms of finite order can be diagonalized.) We write $\lambda_{x}, \lambda_{y}, \lambda_{z}, \lambda_{w}$ for the associated eigenvalues, so that $\sigma^{\prime}$ is given by $(x, y, z, w) \rightarrow$ 
$\left(\lambda_{x} x, \lambda_{y} y, \lambda_{z} z, \lambda_{w} w\right)$. The divisors of $\mathbb{P}$ defined by the above coordinates, and their restrictions to $X$, will be respectively denoted by $P_{x}, P_{y}, P_{z}, P_{w}$ and $C_{x}, C_{y}, C_{z}, C_{w}$. Note that the last four divisors are invariant elements in $\left|-a K_{X}\right|$, for opportune values of $a$.

To simplify the statement of the following proposition, we rename $x, y, z, w$ by $x_{0}, \ldots, x_{3}$, and put $a_{i}=\operatorname{deg} x_{i}$. We write $\lambda_{i}$ for the eigenvalue associated to $x_{i}$. We denote by $P_{i}$ the divisor on $\mathbb{P}$ defined by $x_{i}$, and set $C_{i}=P_{i} \cap X$.

Proposition 3.2.2. Assume that, for some value of $i, C_{i}$ is a fixed divisor of $X$ (that is, every point of $C_{i}$ is fixed). Then there is an opportune choice of $\sigma^{\prime}$ such that $\lambda_{j}=1$ for all $j \neq i$.

Proof. Up to renaming the coordinates, we can assume that $i=0$. We can find a point $p \in C_{0}$ whose coordinates $\left(0, x_{1}, x_{2}, x_{3}\right)$ satisfy $x_{1} x_{2} x_{3} \neq 0$. Since $\sigma(p)=p$, we have $\lambda_{1}^{1 / a_{1}}=\lambda_{2}^{1 / a_{2}}=\lambda_{3}^{1 / a_{3}}$. We can also assume that $a_{1}=1$, and choose $\sigma^{\prime}$ such that $\lambda_{1}=1$ (this can be done by replacing $\lambda_{i}$ with $\left.\lambda_{i} \lambda_{1}^{-a_{i}}\right)$. We deduce then that $\lambda_{2}=\lambda_{3}=1$, too.

Clearly, the choice of $\sigma^{\prime}$ as in the statement of Proposition 3.2.2 is unique. We can view this condition as a way of fixing a distinguished action of $\sigma$ on $S$. In fact, if $s \in S$ is the homogeneous element defining $X$, so that $X=\operatorname{Proj} S /(s)$, then we also get a distinguished action of $\sigma$ on $S /(s)$. Therefore, when the assumptions of the proposition above are satisfied, we shall use $\sigma$ to denote these particular automorphisms of $S$ and $S /(s)$. Recall that $S /(s)$ is isomorphic to the anticanonical ring of $X$. It is important to keep in mind that the above "distinguished" action on $S$ is not necessarily the one induced, via this isomorphism, by the natural action on the anticanonical ring.

\subsection{Automorphisms of prime order of plane cubic curves}

Let $C$ be an irreducible plane cubic curve and $\sigma$ an automorphism of $C$ of prime order $n \geq 3$. Let $f: C \rightarrow C / \sigma$ be the projection on the quotient and $R \subset C$ be the fixed point set. If $C$ is singular, let $q$ denote the singular point.

Proposition 3.3.1. If $C$ is smooth, then either $R=\emptyset$ and $C / \sigma$ is an elliptic curve (any value of $n$ may occur), or $R \neq \emptyset, C / \sigma \cong \mathbb{P}^{1}$ and $n=3$. If $C$ is a nodal cubic, then $q$ is the only fixed point and $C / \sigma$ is 
isomorphic to a nodal cubic: the two tangent directions at $q$ are fixed by $\sigma$, and the corresponding eigenvalues are $\lambda$ and $\lambda^{-1}$, where $\lambda \neq 1$ is a $n^{\text {th }}$-root of unity. If $C$ is a cuspidal cubic, then there is another fixed point distinct from $q$ and $C / \sigma \cong \mathbb{P}^{1}$.

Proof. The first case follows directly by Hurwitz formula. So, assume that $C$ is singular. Clearly $q$ is a fixed point. Take the normalization $\mathbb{P}^{1} \rightarrow C$. Then $\sigma$ lifts to a (non-trivial) automorphism of $\mathbb{P}^{1}$. We start considering the case when $q$ is a node of $C$. Let $q^{\prime}, q^{\prime \prime} \in \mathbb{P}^{1}$ be the two inverse images of $q$. Since $n$ is odd, $q^{\prime}$ and $q^{\prime \prime}$ are necessarily fixed points. Therefore $q$ is the only fixed point of $C$ and, locally, the two branches of $C$ passing through $q$ are stabilized by $\sigma$. By expressing $\sigma$ in two affine charts of $\mathbb{P}^{1}$, we determine the two eigenvalues of the action induced on $T_{q} C$. Then the computation of the invariant subring of the local ring of $X$ at $q$ shows that $C / \sigma$ has an ordinary node at $f(q)$. Now suppose that $q$ is a cuspidal point. By considering the action on $\mathbb{P}^{1}$, we see that there is another fixed point beside $q$. This time the local computation shows that $C / \sigma$ is smooth at $f(q)$.

\subsection{Equivariant Mori theory}

Let $X$ be a smooth projective variety and $G$ be a finite group acting on $X$. Then $G$ acts on $N^{1}(X)$ and $N_{1}(X)$, and the perfect pairing ( · ) : $N^{1}(X) \times N_{1}(X) \rightarrow \mathbb{R}$ restricts to a perfect pairing $N^{1}(X)^{G} \times N_{1}(X)^{G} \rightarrow \mathbb{R}$. In particular, $N^{1}(X)^{G}$ and $N_{1}(X)^{G}$ have the same dimension, that we shall denote by $\rho(X)^{G}$.

Assume that $\rho(X)^{G} \geq 2$. The cone $\overline{\mathrm{NE}}(X)^{G}:=\overline{\mathrm{NE}}(X) \cap N_{1}(X)^{G}$ is called $G$-invariant cone of curves of $X$. Let $F^{G}$ be a negative extremal face of $\overline{\mathrm{NE}}(X)^{G}$. First of all, note that $F^{G}$ is contained in the boundary of $\overline{\mathrm{NE}}(X)$. Let $F$ be the smallest extremal face of $\overline{\mathrm{NE}}(X)$ containing $F^{G}$.

Proposition 3.4.1. $F$ is invariant under the action of $G$, and the extremal contraction $\operatorname{cont}_{F}: X \rightarrow Y$ of the face $F$ is a G-equivariant morphism.

Proof. Let $L_{F}$ be a good-supporting divisor for the face $F$, and consider the divisor $L=\sum_{g \in G} g L_{F} . L$ is still nef, is positive on $\overline{\mathrm{NE}}(X)-F$, and vanishes along $F^{G}$. Therefore $L$ is a good-supporting divisor for some extremal face $F^{\prime}$ of $\overline{\mathrm{NE}}(X)$ with $F^{G} \subseteq F^{\prime} \subseteq F$. By assumption on the dimension of $F$, we conclude that $F^{\prime}=F$. Since $L$ is $G$-equivariant, so are $F$ and $\operatorname{cont}_{F}$. 


\subsection{Resolution of indeterminacy of pairs}

Let $\psi: X^{\prime} \rightarrow X$ be a birational map between two projective varieties. If $G$ is a subgroup of $\operatorname{Bir}(X)$ and $G^{\prime}=\psi^{-1} G \psi$, then we say that $(X, G)$ and $\left(X^{\prime}, G^{\prime}\right)$ are birationally equivalent pairs. If in addition $X^{\prime}$ is smooth and $G^{\prime} \subseteq \operatorname{Aut}\left(X^{\prime}\right)$, then we say that $\left(X^{\prime}, G^{\prime}\right)$ is a resolution of indeterminacy of the pair $(X, G)$. We recall the following result (see [11] for a proof and a stronger statement for the case of smooth surfaces).

TheOREM 3.5.1. In the notation above, assume that $G$ is finite. Then there exists a resolution of indeterminacy $\left(X^{\prime}, G^{\prime}\right)$ of the pair $(X, G)$.

\section{§4. Proofs of Theorems A and B}

Let $X$ be a smooth projective surface whose canonical class is not nef, and let $\sigma \in \operatorname{Aut}(X)$ be an element of finite order.

LEMMA 4.0.2. The pair $(X, \sigma)$ is minimal if and only if for each $(-1)$ curve $E$ of $X$ there exists an integer $k$ such that $E$ and $\sigma^{k} E$ intersect properly.

Proof. Suppose that there is a (-1)-curve $E$ such that, for some $m \geq 1$, $\sigma^{k} E \cap E=\emptyset$ for $1 \leq k \leq m-1$ and $\sigma^{m} E=E$. Then the contraction of the disjoint $(-1)$-curves $\sigma^{k} E$, for $k=0, \ldots, m-1$, gives an equivariant birational morphism onto a smooth surface, so $(X, \sigma)$ is not minimal. Conversely, let $f: X \rightarrow X^{\prime}$ be a non-trivial birational morphism of smooth surfaces, $\sigma^{\prime} \in \operatorname{Aut}\left(X^{\prime}\right)$ be an element of finite order, and $\sigma \in \operatorname{Aut}(X)$ be such that $f \sigma=\sigma^{\prime} f$. Let $E$ be a $(-1)$-curve contained in the exceptional locus of $f$ and $m$ the least positive integer such that $\sigma^{m} E=E$. Note that the curve $C=E+\sigma E+\cdots+\sigma^{m-1} E$ is $f$-exceptional, so $C^{2}<0$. Suppose that $E$ and $\sigma^{k} E$ meet properly for some positive $k \leq m-1$. Then for every $i=0, \ldots, m-1$, if $j(i)$ denotes the least non-negative integer such that $i+k \equiv j(i)$ modulo $m$, we have $\sigma^{i} E \cdot \sigma^{j(i)} E \geq 1$. Note that $j(i) \neq i$. Then we get the contradiction

$0>C^{2}=\sum_{i=0}^{m-1} \sum_{j=0}^{m-1} \sigma^{i} E \cdot \sigma^{j} E \geq \sum_{i=0}^{m-1}\left(\left(\sigma^{i} E\right)^{2}+\sigma^{i} E \cdot \sigma^{j(i)} E\right) \geq-m+m \geq 0$.

\subsection{The invariant part of the cone of curves}

Assume that $K_{X}$ is not nef, $\sigma$ has prime order $n$, and $(X, \sigma)$ is a minimal pair. Recall that $\rho(X)^{\sigma}$ denotes the rank of $\operatorname{NS}(X)^{\sigma}$. 
Proposition 4.1.1. Suppose that $\rho(X)^{\sigma} \geq 2$. Then $(X, \sigma)$ is one of cases 2 and 3 of Theorem A.

Proof. Since $\rho(X)^{\sigma} \geq 2$ and $K_{X}$ is not nef, we can find an extremal ray $R$ of $\overline{\mathrm{NE}}(X)^{\sigma}$ contained in the negative part of $\partial \overline{\mathrm{NE}}(X)$. Let $F$ be the smallest extremal face of $\overline{\mathrm{NE}}(X)$ containing $R$. By Proposition 3.4.1, the contraction of $F$ is $\sigma$-equivariant. We claim that either $F=R$, or $n=2$ and the extremal rays of $F$ are generated by $(-1)$-curves $E$ satisfying $E \cdot \sigma E=1$. To see this, suppose that $F \neq R$. Let $[E]$ be a minimal generator of an extremal ray of $F$ and consider the curve $C=E+\sigma E+\cdots+\sigma^{n-1} E$. Note that $[C] \in \partial \overline{\mathrm{NE}}(X)$, so $C^{2} \leq 0$ by [25, Lemma 2.5]. If $n=2$, then

$$
0 \geq C^{2}=E^{2}+2 E \cdot \sigma E+(\sigma E)^{2}=2\left(E^{2}+E \cdot \sigma E\right) .
$$

We observe that the possibility $E^{2}=E \cdot \sigma E=0$ can not occur, since $[E]$ and $[\sigma E]$ span different rays. Thus $E^{2}=-1$ and $E \cdot \sigma E=1$, as claimed. Consider now the case $n \geq 3$. First of all, note that the $\sigma^{i} E$ are $(-1)$-curves. By Lemma 4.0.2, there is a positive integer $k$ such that $E \cdot \sigma^{k} E \geq 1$. We put $\eta=\sigma^{k}$. Note that $\eta$ has order $n$, and $(X, \eta)$ is a minimal pair. We deduce that $\eta^{i} E \cdot \eta^{j} E \geq 0$ for $0 \leq i<j \leq n-1$, and in fact $\eta^{i} E \cdot \eta^{i+1} E \geq 1$ for all $i$. Note also that $C=E+\eta E+\cdots+\eta^{n-1} E$. Keeping in mind that $n \geq 3$, we get the contradiction

$$
0 \geq C^{2} \geq \sum_{i=0}^{n-1}\left(\left(\eta^{i} E\right)^{2}+2 \eta^{i} E \cdot \eta^{i+1} E\right) \geq-n+2 n>0 .
$$

Now we can conclude the proof of Proposition 4.1.1. First, suppose that $R=F$. Since $(X, \sigma)$ is a minimal pair, the $\sigma$-equivariant contraction of $F$ is a $\mathbb{P}^{1}$-bundle fibration. If $\sigma$ acts on each fiber, then there are not fixed fibers since the set of fixed points of $X$ is smooth and contains a double section. This gives case 2 of Theorem A. Now suppose that $R \subsetneq F$. By the above arguments, the $\sigma$-equivariant contraction of $F$ is a conic bundle whose singular fibers are curves of the form $E \cup \sigma E$. Let $q$ be the singular point of a singular fiber. Since the action on $\mathrm{T}_{q} X$ has eigenvalues 1 and -1 , we can find, locally near $q$, a fixed section through $q$. This implies that the automorphism induced on $Y$ is trivial. The same argument used for the case of $\sigma$-invariant $\mathbb{P}^{1}$-bundles shows that $\sigma$ restricts to an effective action on each fiber. This gives case 3 of Theorem A.

Proposition 4.1.2. Suppose that $X \cong \mathbb{P}^{1} \times \mathbb{P}^{1}$ and $\rho(X)^{\sigma}=1$. Then $n=2$ and $\sigma$ swaps the two rulings of $X$. This is case 4 of Theorem A. 
Proof. Since $\rho(X)^{\sigma}=1, n=2$ and $\sigma$ swaps the two extremal rays of $\overline{\mathrm{NE}}(X)$.

Definition 4.1.3. Let $X$ be a smooth Del Pezzo surface. The set of $(-1)$-curves of $X$ splits in orbits under the action of $\sigma$. We call orbits of $(-1)$-curves the divisors on $X$ of the form $D=E+\sigma E+\cdots+\sigma^{n-1} E$, with $E$ a $(-1)$-curve of $X$.

Proposition 4.1.4. Suppose that $X \neq \mathbb{P}^{2}, \mathbb{P}^{1} \times \mathbb{P}^{1}$ and $\rho(X)^{\sigma}=1$. Then either $n=2$ and $X$ is a Del Pezzo surface of degree 1 or 2 , or $n=3$ and $X$ is a Del Pezzo surface of degree 1 or 3 , or $n=5$ and $X$ is a Del Pezzo surface of degree 1 or 5.

Proof. By pulling back an ample line bundle from $X / \sigma$, we see that one of the two generators of $\operatorname{NS}(X)^{\sigma} \cong \mathbb{Z}$ is ample. Since $K_{X}$ is in $\operatorname{NS}(X)^{\sigma}$ and is not nef, $-K_{X}$ is ample, that is, $X$ is a Del Pezzo surface. Then $X \cong \mathrm{Bl}_{\Sigma} \mathbb{P}^{2}$ where $\Sigma \subset \mathbb{P}^{2}$ is a set of $r$ distinct points in general position, $1 \leq r \leq 8$. We recall that the degree of $X$ as Del Pezzo surface is $d=K_{X}^{2}=9-r$. Let $D$ be an orbit of $(-1)$-curves of $X$. Since $D$ is invariant under the action of $\sigma$, $D \in\left|-a K_{X}\right|$ for some positive integer $a$. Then $n=D \cdot\left(-K_{X}\right)=a d$, hence $d=1$ or $n$, since $n$ is prime. We can conclude by the fact that $n$ divides the number of $(-1)$-curves of $X$ (this number is well known as a function of $d$, see for instance [23]).

The remaining part of this section is devoted to the analysis of the six cases presented by Proposition 4.1.4.

\subsection{The Geiser and Bertini involutions}

Let $\sigma$ be a biregular involution on a Del Pezzo surface $X$ of degree 1 or 2 , and assume that $\rho(X)^{\sigma}=1$. Then the action that $\sigma$ induces on $\operatorname{Pic}(X)$ is the same as the one induced by the Bertini or the Geiser involution, respectively. Since their difference induces an automorphism of $\mathbb{P}^{2}$ which fixes the points of $\Sigma$, they are the same automorphism.

\subsection{Cases $\mathrm{A} 1$ and $\mathrm{B} 1$}

Let $X$ be a smooth Del Pezzo surface of degree 3 and $\sigma$ be an automorphism of $X$ of order 3 such that $\rho(X)^{\sigma}=1$.

Proposition 4.3.1. Let $X$ and $\sigma$ as above. Then $(X, \sigma)$ is as in case $\mathrm{A} 1, X / \sigma \cong \mathbb{P}^{2}$, and the quotient map $f: X \rightarrow X / \sigma$ is totally ramified over a smooth plane cubic. Moreover, $f$ is defined by the linear subsystem of $\left|-K_{X}\right|$ spanned by the orbits of $(-1)$-curves. 
Proof. We identify $X$ with its anticanonical embedding in $\mathbb{P}^{3}$. Since $\sigma$ acts on $\left|-K_{X}\right|, \sigma$ is the restriction to $X$ of a linear automorphism of $\mathbb{P}^{3}$. In particular, the fixed point set $R$ consists of points, lines and possibly a smooth plane cubic. Since $\rho(X)^{\sigma}=1$, the eigenvalues of the action of $\sigma$ on $H^{2}(X, \mathbb{Z})$ are 1 , three times $\lambda$, and three times $\lambda^{2}$, with $\lambda=e^{2 \pi i / 3}$, thus the trace is -2 . Therefore the trace of $\sigma$ acting on $H^{*}(X, \mathbb{Z})$ is 0 . By Lefschetz this is the sum of the Euler numbers of the fixed components of $\sigma$. We conclude that $R$ is a plane section of $X$, hence $\sigma$ fixes a plane in $\mathbb{P}^{3}$. It follows at once that $X$ has equation of the form $x^{3}=F(y, z, w)$ and $f$ is the projection over the plane $x=0$. Since the orbits of $(-1)$-curves are mapped to lines of this $\mathbb{P}^{2}$ spanning $\left|\mathcal{O}_{\mathbb{P}^{2}}(1)\right|, f$ is defined by the claimed linear system.

\subsection{Cases A2 and B2}

Let $X$ be a Del Pezzo surface of degree 1 and $\sigma$ be an automorphism of $X$ of order 3 such that $\rho(X)^{\sigma}=1$. As usual, $f$ will denote the quotient map. Note that the base point $q$ of $\left|-K_{X}\right|$ is fixed by $\sigma$. In particular, $\sigma$ lifts to an automorphism of $Y=\mathrm{Bl}_{q} X$ which stabilizes the exceptional divisor $E_{q}$. We denote by $g: Y \rightarrow Y / \sigma$ the quotient map.

Lemma 4.4.1. The action induced by $\sigma$ on $\left|-K_{X}\right|$ is trivial.

Proof. Arguing as in the proof of Proposition 4.3.1, we see that the trace of $\sigma$ acting on $H^{*}(X, \mathbb{Z})$ is -1 . If $\sigma$ does not act trivially on $\left|-K_{X}\right|$ the fixed components are points or smooth curves of genus $\leq 1$. Since these have positive Eulen number, this is impossible.

Lemma 4.4.2. The ramification locus $R$ of $f$ is the disjoint union of $q$ and $C_{z}$ (see Notations 3.2.1). Moreover, $f(q)$ is a singularity of $X / \sigma$ of type $\frac{1}{3}(1,1)$.

Proof. It follows from Lemma 4.4.1 that $E_{q}$ is a fixed curve on $Y$. Since fixed divisors are smooth, we deduce that $q$ is an isolated fixed point of $X$. $f(q)$ is a singularity of type $\frac{1}{3}(1,1)$ because $\sigma$ acts trivially on $\mathbb{P} T_{q} X$. Let $R_{1}$ be the union of the 1-dimensional components of $R$. Since $R$ intersects the general $C \in\left|-K_{X}\right|$ in three points, one of which is $q$, we have $R_{1} \cdot C=2$. Noting that $R_{1}$ is invariant and $\operatorname{Pic}(X)^{\sigma}=-K_{X} \mathbb{Z}$, we deduce that $R_{1} \in$ $\left|-2 K_{X}\right|$. Since $R_{1}$ intersects properly every $C \in\left|-K_{X}\right|$, it must be $C_{z}$. By Lefschetz, the Euler number of $R$ is -1 . Since $C_{z}$ has Euler number -2 , we conclude that $q$ is the only isolated fixed point. 
Proposition 4.4.3. Let $X$ be a smooth Del Pezzo surface of degree 1 and $\sigma$ be an element of order 3 in $\operatorname{Aut}(X)$. Then $(X, \sigma)$ is as in case A2.

Proof. We can embed $X$ as a hypersurface of degree 6 in the weighted projective space $\mathbb{P}(1,1,2,3)$. We fix coordinates $(x, y, z, w)$ according to Notation 3.2.1. Note that $C_{z}$ is a fixed divisor on $X$. Then Proposition 3.2.2 implies that $\sigma$ is the restriction to $X$ of the automorphism of $\mathbb{P}(1,1,2,3)$ given by $(x, y, z, w) \rightarrow(x, y, \lambda z, w)$, where $\lambda \neq 1$ is a $3 d r$-root of unity. In particular, we deduce that $X$ is defined by an equation of the form $z^{3}=F(x, y, w)$.

We deduce the following

COROLlary 4.4.4. $f$ is the restriction of the linear projection of $\mathbb{P}(1,1,2,3)$ from the point $(0,0,1,0)$ to $P_{z}$ (see Notation 3.2 .1$)$. In particular, $X / \sigma=P_{z} \cong \mathbb{P}(1,1,3) \cong \overline{\mathbb{F}}_{3} \subset \mathbb{P}^{4}$, the cone over a rational twisted cubic. In terms of linear systems, $f$ is defined by the linear subsystem of $\left|-3 K_{X}\right|$ spanned by $3 C_{x}, 2 C_{x}+C_{y}, C_{x}+2 C_{y}, 3 C_{y}, C_{w}$.

If we embed $X$ in $\mathbb{P}^{6}$ by $\left|-3 K_{X}\right|$, then the orbits of $(-1)$-curves are hyperplanes sections, the quotient map $f$ is the restriction to $X$ of a linear projection $\pi: \mathbb{P}^{6} \rightarrow \mathbb{P}^{4}$, and $X / \sigma=\overline{\mathbb{F}}_{3} \subset \mathbb{P}^{4}$. Let $\phi: \mathbb{F}_{3} \rightarrow \overline{\mathbb{F}}_{3}$ be the resolution of the singularity $p \in \overline{\mathbb{F}}_{3}$, and $E=\phi^{-1}(p)$ be the exceptional curve. Then $Y / \sigma=\mathbb{F}_{3}, g\left(E_{q}\right)=E$ and $\phi g=f \psi$. Let $R^{\prime}$ and $B^{\prime}$ be the ramification and branch loci of $g$. Note that they are divisors containing respectively $E_{q}$ and $E$, and that $g^{*} B^{\prime}=3 R^{\prime}$. Using $2 R^{\prime}=K_{Y}-g^{*} K_{\mathbb{F}_{3}}$, one sees that $B^{\prime} \sim 2 \phi^{*} \mathcal{O}_{\overline{\mathbb{F}}_{3}}(1)+E$. Therefore $f$ is branched along the vertex $p$ of $\overline{\mathbb{F}}_{3}$ and a (smooth) curve $B_{1} \in\left|\mathcal{O}_{\overline{\mathbb{F}}_{3}}(2)\right| . \quad B_{1}$ is a sextic and $3 K_{B_{1}} \cong$ $\left.\mathcal{O}_{\mathbb{P}^{4}}(1)\right|_{B_{1}}$ by adjunction, so $B_{1}$ has genus 2 . Riemann-Roch formula implies that the embedding of $B_{1}$ in $\mathbb{P}^{4}$ is given by the complete linear system $\left|K_{B_{1}}\right|$. This concludes the proof of B2.

\subsection{Cases A3 and B3}

Let $X$ be a Del Pezzo surface of degree 1 and $\sigma$ be an automorphism of $X$ of order 5 .

Lemma 4.5.1. $\sigma$ acts effectively on $\left|-K_{X}\right|$, whose invariant curves are a rational cuspidal curve $C_{x}$ and an elliptic curve $C_{y}$ (see Notations 3.2.1). The ramification locus of $f: X \rightarrow X / \sigma$ is the disjoint union of $C_{y}$ and the cuspidal point $q_{1}$ of $C_{x}$. The quotient surface $X / \sigma$ has a unique singularity at the point $f\left(q_{1}\right)$. 
Proof. Since the base point $q$ of $\left|-K_{X}\right|$ is fixed, every invariant but not fixed $C \in\left|-K_{X}\right|$ must be a cuspidal curve by Proposition 3.3.1. We deduce that $\sigma$ acts effectively on $\left|-K_{X}\right|$, so that $C_{x}$ and $C_{y}$ are the only two invariant members of $\left|-K_{X}\right|$. By computing the Euler number of $X-\left(C_{x} \cup C_{y}\right)$, we deduce that one of the two curves, say $C_{y}$, is fixed, while the other one, $C_{x}$, is a rational cuspidal curve. Note that the cuspidal point $q_{1}$ of $C_{x}$ is the only isolated fixed point of $X$, so $f\left(q_{1}\right)$ is the only singularity of $X / \sigma$.

Proposition 4.5.2. Let $X$ be a smooth Del Pezzo surface of degree 1 and $\sigma$ be an element of order 5 in $\operatorname{Aut}(X)$. Then $(X, \sigma)$ is as in case A3.

Proof. The proof is analogous to that of Proposition 4.5.2. This time, in the embedding of $X$ in $\mathbb{P}(1,1,2,3), \sigma$ is the restriction of an automorphism acting effectively only on the $y$ coordinate, and $X$ has equation of the form $x y^{5}=F(x, z, w)$.

Proposition 4.5.3. $X / \sigma$ can be identified with the sextic hypersurface of equation $x u=F(x, z, w)$ in the weighted projective space $\mathbb{P}(1,2,3,5)$ with coordinates $(x, z, w, u)$. Let $p_{1}=(0,1,0,0) \in \mathbb{P}(1,2,3,5)$. Then $p_{1}=f\left(q_{1}\right)$ and is a singularity of $X / \sigma$ of type $\frac{1}{5}(1,4)$. In terms of linear systems, $f$ is defined by the linear subsystem of $\left|-5 K_{X}\right|$ spanned by $5 C_{x}, 5 C_{y}, 3 C_{x}+$ $C_{z}, 2 C_{x}+C_{w}, C_{z}+C_{w}$.

Proof. Since the relation $x y^{5}-F=0$ involves only the 5 th power of $y$, the ring of invariants $T^{\sigma}$ of $T=\mathbb{C}[x, y, z, w] /\left(x y^{5}-F\right)$ is generated, over $\mathbb{C}$, by the classes of $x, y^{5}, z, w$. This yields the projective description of $X / \sigma$, since $T^{\sigma} \cong \mathbb{C}[x, z, w][u]$ with $u=F(x, z, w) / x$. Clearly $p_{1}=f\left(q_{1}\right)$. The equation of $X / \sigma$ near $p_{1}$ is approximated by the linear equation $x=0$ (of weight 1$)$, so the nature of the point $p_{1} \in X$ is the same as the one of the point $(0,0,1)$ of $\mathbb{P}(2,3,5)$. This is a singularity of type $\frac{1}{5}(1,4)$. The last statement of the proposition follows from the fact that $T_{5}^{\sigma}$ generates the ring $\oplus_{m \geq 0} T_{5 m}^{\sigma}$.

We can also follow a more geometric approach to study the quotient $X \rightarrow X / \sigma$, by understanding explicitly the system $\left|-K_{X}\right|$ in terms of the embedding of $X$ in $\mathbb{P}(1,1,2,3)$. For $t \in \mathbb{C}$, we put $C_{t}=C_{y}-t C_{x}$. Note that $C_{x}$ is given by $F(0, z, w)=0$ in $P_{x}$, and $C_{t}$ by $F(x, z, w)=t^{5} x^{6}$ in $P_{t}$. Here $P_{t} \subset \mathbb{P}(1,1,2,3)$ is the subspace defined by $y=t x$. If $L=P_{x} \cap P_{y}$, then $C_{x} \cap L=C_{y} \cap L=q$. We see that $q \neq(0,0,1,0),(0,0,0,1)$ since 
$H^{0}\left(-2 K_{X}\right)$ and $H^{0}\left(-3 K_{X}\right)$ are globally generated. Moreover, we observe that $(0,1,0,0)$ is the cuspidal point $q_{1}$ of $C_{x}$.

Note that either $j\left(C_{t}\right) \neq 0$ or $j\left(C_{t}\right)=0$ for all smooth $C_{t}$. Indeed the restrictions of $x, z, w$ to $C_{t}$ define divisors on $C_{t}$ which are linearly equivalent to $q, 2 q, 3 q$, respectively. Thus, by embedding $C_{t}$ in $\mathbb{P}^{2}$ via $\left|\mathcal{O}_{C_{t}}(3 q)\right|, C_{t}$ is defined, in an affine chart, by $F(1, z, w)=t^{5}$. Writing this equation in Weierstrass normal form $\left(w^{\prime}\right)^{2}=\left(z^{\prime}\right)^{3}+A z^{\prime}+B$, we see that $A$ is independent of $t$.

Let $\pi: \mathbb{P}(1,1,2,3) \rightarrow P_{y}$ be the linear projection from the point $q_{1}=$ $(0,1,0,0)$. Its restriction to $X$ contracts $C_{x}$ to $q$ and coincides with $f$ outside $C_{x}$, since $\left.\pi\right|_{\left(X-C_{x}\right)}$ is finite of degree 5 and $\sigma$ acts on the fibers of $\pi$. We resolve the indeterminacy of $\pi$ by taking the weighted blowup of $\mathbb{P}(1,1,2,3)$ at $q_{1}=(0,1,0,0)$, with weights $1,2,3$. This restricts to the weighted blowup $g: \mathrm{Bl}_{q_{1}}^{w} X \rightarrow X$ of $X$ at $q_{1}$ with weights 2,3 . The latter gives a resolution $f_{1}: \mathrm{Bl}_{q_{1}}^{w} X \rightarrow P_{y}$ of $\left.\pi\right|_{X}$. Let $E_{1}$ be the exceptional divisor of $g$ and $C_{x}^{\prime}, C_{y}^{\prime}$ be the strict transforms of $C_{x}, C_{y}$. Next we take the blowup $\mathrm{Bl}_{q} P_{y}$ of $P_{y}$ at $q$. Let $E$ and $L^{\prime}$ be the corresponding exceptional divisor and the strict transform of $L$. Then $f_{1}$ factors through a morphism $f_{2}: \mathrm{Bl}_{q_{1}}^{w} X \rightarrow \mathrm{Bl}_{q} P_{y}$ and $\mathrm{Bl}_{q} P_{y} \rightarrow P_{y}$, and, moreover, $f_{2}\left(C_{x}^{\prime}\right)=E$ and $f_{2}\left(E_{1}\right)=L^{\prime}$. Indeed $f_{1}^{-1}(q)=C_{x}^{\prime}$, and this curve intersects $E_{1} \cong \mathbb{P}(2,3)$ at a point distinct from $(1,0)$ and $(0,1)$. So $\mathrm{Bl}_{q_{1}}^{w} X$ is smooth along this curve, and we can apply the universal property of the blowup to $f_{1}$. Moreover, $f_{2}\left(C_{x}^{\prime}\right)=E$, since $C_{x}^{\prime}$ is the only curve contracted by $f_{1}$, and, by construction, $f_{1}\left(E_{1}\right)=P_{x} \cap P_{y}=L$, so $f_{2}\left(E_{1}\right)=L^{\prime}$.

Proposition 4.5.4. There is a morphism $g^{\prime}: \mathrm{Bl}_{q} P_{y} \rightarrow X / \sigma$ such that $f g=g^{\prime} f_{2}$. Moreover, $X / \sigma=\nu(Z)$, where $Z \cong Z_{22}\left(\cong Z_{211}\right)$ if $j(C)=0$ $\left(\neq 0\right.$, respectively) for some (equivalently, for every) smooth $C \in\left|-K_{X}\right|$, and $\nu$ is the contraction of $G^{\prime} \sqcup S_{0}$ to $p \sqcup p_{1}$ (see Notation 2.0.6).

Proof. Let $h: \widetilde{P}_{y} \rightarrow P_{y}$ be the minimal resolution of the two singularities of $P_{y}$. Note that $6 L$ is a Cartier divisor, and $h^{*}(6 L)=6 \widetilde{L}+$ $3 F+4 H+2 H^{\prime}$. Here $\widetilde{L}=h_{*}^{-1} L, F=h^{-1}((0,1,0))$ is a $(-2)$-curve, and $H \cup H^{\prime}=h^{-1}((0,0,1))$ is a chain of two $(-2)$-curves. We compute $\widetilde{L}^{2}=-1$ by $L^{2}=1 / 6$. Let $\widetilde{q}=h^{-1}(q)$. Then $h$ lifts to a morphism $h^{\prime}: \mathrm{Bl}_{\widetilde{q}} \widetilde{P}_{y} \rightarrow \mathrm{Bl}_{q} P_{y}$, and the exceptional divisor $\widetilde{E}$ of $\mathrm{Bl}_{\widetilde{q}} \widetilde{P}_{y}$ is mapped isomorphically to $E$. The strict transform, over $\mathrm{Bl}_{q} \widetilde{P}_{y}$, of the curve $\widetilde{L} \cup F \cup H \cup H^{\prime}$ is a chain of four $(-2)$-curves. Its contraction $\widetilde{g}: \mathrm{Bl}_{\widetilde{q}} \widetilde{P}_{y} \rightarrow X^{\prime}$ 
factors through $h^{\prime}$ and a morphism $g^{\prime}: \mathrm{Bl}_{q} P_{y} \rightarrow X^{\prime}$ which contracts $L^{\prime}$ (generating a singularity of type $\frac{1}{5}(1,4)$ ) and is an isomorphism outside $L^{\prime}$. This gives $g^{\prime} f_{2} g^{-1}=f$, hence $X^{\prime}=X / \sigma$.

Let $D_{t}=\left(\left.\pi\right|_{X}\right)_{*} C_{t}$, for $t \in \mathbb{C}$. The minimal smooth resolution $\nu: Z \rightarrow$ $X / \sigma$ of the base locus of $f_{*}\left|-K_{X}\right|$ (which is supported at $p$ ) factors as $\nu=\widetilde{\nu} \widetilde{g} . Z$ is an elliptic fibration with only one section $S_{0}$ (the exceptional divisor of the last monoidal transformation) and having a singular fiber $F_{0}$ of type $I I^{*}$, so it is either $Z_{22}$ or $Z_{211}$ accordingly to the $j$-invariant of the elements in $\left|-K_{X}\right|$. We conclude by observing that $\nu$ is the contraction of $G^{\prime} \sqcup S_{0}$, where $G^{\prime}$ is as in Notation 2.0.6.

To conclude the proof of B3, we observe that the branch divisor of $f$ is the proper transform of $C_{y} \subset P_{y}$, so it is an elliptic curve.

\subsection{Cases A4 and B4}

Let $X$ be the Del Pezzo surface of degree 5 and $\sigma$ be an automorphism of $X$ of order 5 . Although it is known that $\operatorname{Aut}(X) \cong S_{5}$, the symmetric group on five letters, we will explicitly tread also this case. In a suitable coordinate system, $\mathrm{X}$ is the blowup of $\mathbb{P}^{2}$ along the set $\Sigma$ of four points $p_{i}$ in general position. Let $L_{i, j}$ be the line passing through the points $p_{i}$ and $p_{j}, L_{i, j}^{\prime}$ be the proper transform of $L_{i, j}$ over $X, E_{i}$ be the exceptional divisor over the point $p_{i}$. The set $\left\{L_{i, j}^{\prime}, E_{i}\right\}_{i, j}$ is the set of $(-1)$-curves of $X$. One can check that none of the $(-1)$-curves can be invariant and the five components of each $\sigma$-orbit of $(-1)$-curves are configured into a pentagon. We can assume, without lost of generality, that the two orbits are $D_{1}=L_{1,2}^{\prime}+E_{1}+L_{1,4}^{\prime}+L_{2,3}^{\prime}+E_{2}$ and $D_{2}=L_{3,4}^{\prime}+L_{1,3}^{\prime}+E_{4}+E_{3}+L_{2,4}^{\prime}$. The five points of intersection of $D_{1}$ and $D_{2}$ establish (in a obvious way) a one-to-one correspondence between the components of $D_{1}$ and $D_{2}$. Fix coordinates $(x, y, z)$ in $\mathbb{P}^{2}$ such that $p_{1}=(1,0,0), p_{2}=(0,1,0), p_{3}=(0,0,1)$ and $p_{4}=(1,1,1)$, and let $\tau$ be the order 5 Cremona transformation defined by

$$
(x, y, z) \rightarrow(x z, x(z-y), z(x-y)) .
$$

Proposition 4.6.1. Let $X$ be the Del Pezzo surface of degree 5 and $\sigma$ be an automorphism of $X$ of order 5 . In the notation above, after suitably reordering the points $p_{i}, \sigma$ is the lift on $X$ of $\tau$. This is case A4.

Proof. By means of the correspondence described above, an order 5 automorphism of $X$ is uniquely determined by the action induced on the 
orbit of one $(-1)$-curve. We deduce that $\tau^{\prime}=\sigma^{m}$ for some $m$. Consider the elements $\eta, \phi, \psi \in \operatorname{Aut}\left(\mathbb{P}^{2}\right)$ determined by the tree permutations $\left(p_{1} p_{4} p_{2} p_{3}\right)$, $\left(p_{1} p_{3} p_{2} p_{4}\right)$ and $\left(p_{1} p_{2}\right)\left(p_{3} p_{4}\right)$ on $\Sigma$, and let $\eta^{\prime}, \phi^{\prime}, \psi^{\prime} \in \operatorname{Aut}(X)$ be their lifts over $X$. Then one can check that $\sigma, \sigma^{2}, \sigma^{3}, \sigma^{4}$ are conjugated one into the other by elements in $\left\{\eta^{\prime}, \phi^{\prime}, \psi^{\prime}\right\}$.

Remark 4.6.2. $\tau, \tau^{2}, \tau^{3}, \tau^{4}$ are conjugated one into the other by elements in $\{\eta, \phi, \psi\}$.

By blowing up $X$ along $D_{1} \cap D_{2}$, we obtain an elliptic fibration $Y \cong$ $Z_{5511}$. The two fibers of type $I_{5}$ are the strict transforms $D_{1}^{\prime}$ and $D_{2}^{\prime}$ of $D_{1}$ and $D_{2}$. Let $F_{1}$ and $F_{2}$ denote the other two singular fibers. Note that $\sigma$ acts fiberwise on $Y$. By Euler number computation and Proposition 3.3.1, we can see that the elliptic fibration is $\sigma$-invariant and the nodes $y_{1}, y_{2}$ of the two fibers $F_{1}, F_{2}$ are the only fixed points of $Y$. Let $g: Y \rightarrow Y / \sigma$ be the quotient map and $\nu: Z \rightarrow Y / \sigma$ be the minimal resolution of singularities.

Proposition 4.6.3. $\quad Y / \sigma$ is an elliptic fibration over $\mathbb{P}^{1}$ having exactly four singular fibers of type $I_{1}$ and two singular points of type $\frac{1}{5}(1,4)$, and $Z \cong Z_{5511}$.

Proof. The elliptic fibration of $Y$ induces a fibration on the quotient, and each $D_{i}^{\prime}$ is mapped to a nodal curve. Proposition 3.3.1, applied to the irreducible fibers of $Y$, yield the first part of the proposition. By resolving the two singularities of $Y / \sigma^{\prime}$, we obtain $Z \cong Z_{5511}$.

If $G_{i}=h_{*}^{-1} g\left(D_{i}^{\prime}\right)$ and $G_{i}^{\prime}=h^{-1} g\left(y_{i}\right) \backslash G_{i}$, we deduce the following

Corollary 4.6.4. $X / \sigma \cong \nu\left(Z_{5511}\right)$, where $\nu$ is the contraction of the cycle $S_{0} \sqcup G_{1}^{\prime} \sqcup G_{2}^{\prime}$. In particular, $X / \sigma$ has two singularities of type $\frac{1}{5}(1,4)$ and $f: X \rightarrow X / \sigma$ is ramified over these two points.

\subsection{The last statement of Theorem $\mathbf{A}$}

Let $X \subset \mathbb{P}(1,1,2,3)$ be a smooth sextic surface and, for $i=2,3$, assume there is a $\sigma_{i} \in \operatorname{Aut}(X)$ such that $\left(X, \sigma_{i}\right)$ is as in case Ai. The quotient map $f_{i}: X \rightarrow X / \sigma_{i}$ is the restriction to $X$ of a linear projection $\pi_{q_{i}}: \mathbb{P}(1,1,2,3) \rightarrow P_{i}$, where $P_{i} \subset \mathbb{P}(1,1,2,3)$ is a suitable subspace. We can assume that $P_{i} \cap X$ is the ramification divisor of $f_{i}$. Then we can choose coordinates $(x, y, z, w)$ in $\mathbb{P}(1,1,2,3)$ such that $q_{2}=(0,0,1,0)$, 
$P_{2}=\{z=0\}, q_{3}=(0,1,0,0)$ and $P_{3}=\{y=0\}$. In this coordinate system, $X$ is defined by an equation of the form $x y^{5}+z^{3}+G(x, w)=0$. Using that $X$ is smooth, one can check that this equation is reduced to $x y^{5}+z^{3}+w^{2}+x^{6}=0$ by an opportune change of coordinates. The proofs of Theorems A and B are now complete.

\section{$\S 5$. Building back the covering}

The following proposition gives the construction of $(X, \sigma)$ (for cases B1B3) starting from the quotient $X / \sigma$.

Proposition C.

C1. Let $B$ be a smooth cubic of $\mathbb{P}^{2}$. Then there is a triple cyclic cover $f: X \rightarrow \mathbb{P}^{2}$ branched along $B$, and $(X, \sigma)$ is as in case A1 for any generator $\sigma$ of the Galois group of the covering.

C2. Let $\overline{\mathbb{F}}_{3} \subset \mathbb{P}^{4}$ be a cone over a rational twisted cubic, and let $B_{1} \subset \overline{\mathbb{F}}_{3}$ be a smooth curve of genus 2 , cut on the cone by a quadric hypersurface of $\mathbb{P}^{4}$. Then there is a triple cyclic cover $f: X \rightarrow \overline{\mathbb{F}}_{3}$ branched along $B_{1}$ and the vertex $p$ of the cone, and $(X, \sigma)$ is as in case $\mathrm{A} 2$ for any generator $\sigma$ of the Galois group of the covering.

C3. Let $Z=Z_{22}$ or $Z_{211}$, and $\nu: Z \rightarrow Y$ be the contraction of $G^{\prime} \cup S_{0}$. Let $B_{1}$ be a smooth member of $\left|-K_{Y}\right|$. Then there is a cyclic cover $f: X \rightarrow Y$ of degree 5 branched along $B_{1}$ and the singular point $p_{1}$ of $Y$, and $(X, \sigma)$ is as in case A3 for any generator $\sigma$ of the Galois group of the covering. In particular $X \cong X_{0}$ if and only if $Z=Z_{22}$.

Proof. Consider case $\mathrm{C} 1$. The section $s \in H^{0}\left(\mathcal{O}_{\mathbb{P}^{2}}(3)\right)$ vanishing along $B$ determines a triple cyclic cover $f: X \rightarrow \mathbb{P}^{2}$, where $X=\operatorname{Spec}\left(\mathcal{O}_{\mathbb{P}^{2}} \oplus\right.$ $\left.\mathcal{O}_{\mathbb{P}^{2}}(-1) \oplus \mathcal{O}_{\mathbb{P}^{2}}(-2)\right) . \quad X$ is smooth and $f$ is totally ramified over $B$. Since $-K_{X}=f^{*} \mathcal{O}_{\mathbb{P}^{2}}(1)$, it is ample and has self intersection $K_{X}^{2}=3$ by projection formula. To conclude, let $\sigma$ be a generator of the Galois group of $X \rightarrow \mathbb{P}^{2}$. Then $\rho(X)^{\sigma}=\rho\left(\mathbb{P}^{2}\right)=1$.

For case $\mathrm{C} 2$, consider the blowup $\phi: \mathbb{F}_{3} \rightarrow \overline{\mathbb{F}}_{3}$ of $\overline{\mathbb{F}}_{3}$ at the vertex $p$. Let $E$ denote the exceptional divisor. Let $H=\phi^{*} \mathcal{O}_{\mathbb{F}_{3}}(1)$ and $\widetilde{B}=$ $\phi^{-1}\left(B_{1} \cup\{p\}\right)$. Then $\widetilde{B} \in|2 H+E|=|3(H-F)|$. Set $\mathcal{L}=\mathcal{O}_{\mathbb{F}_{3}}(H-F)$. The section $s \in H^{0}\left(\mathcal{L}^{3}\right)$ defining $B_{1}$ determines a triple cyclic cover $\widetilde{f}: \widetilde{X} \rightarrow \mathbb{F}_{3}$ (totally) ramified over $\widetilde{B}$, where $\widetilde{X}=\operatorname{Spec}\left(\mathcal{O}_{\mathbb{F}_{3}} \oplus \mathcal{L}^{-1} \oplus \mathcal{L}^{-2}\right)$. $\widetilde{X}$ is smooth since $\mathbb{F}_{3}$ and $\widetilde{B}$ are smooth. Set $E^{\prime}:=\widetilde{f}^{-1}(E)$. Then $E^{\prime}=(1 / 3) \widetilde{f}^{*}(E)$ 
and $\widetilde{f}_{*} E^{\prime}=E$. By projection formula, $\left(E^{\prime}\right)^{2}=-1$, so there is a morphism $\psi: \widetilde{X} \rightarrow X$ contracting $E^{\prime}$ to a smooth point. Then we can find a morphism $f: X \rightarrow \overline{\mathbb{F}}_{3}$ such that $f \psi=\phi \widetilde{f}$. By construction, $f$ is a degree 3 cyclic cover branched along $B$. Since $-K_{X}=(1 / 3) f^{*} H_{\bar{F}_{3}},-K_{X}$ is ample. Moreover, $H_{\mathbb{F}_{3}}^{2}=3$ yields $K_{X}^{2}=1$ by projection formula. To conclude, let $\sigma$ be one of the two generators of the Galois group of $X \rightarrow \overline{\mathbb{F}}_{3}$. Then $\rho(X)^{\sigma}=\rho\left(\overline{\mathbb{F}}_{3}\right)=$ 1.

For case C3, set $C=\nu(G)$ ( $G$ is the irreducible component of the fiber $F_{0}$ occurring with multiplicity 5 , and is the only component not contracted by $\nu$ ). Then $B_{1}$ is linearly equivalent to $5 C$. The section $s \in H^{0}\left(\mathcal{O}_{Y}(-5 C)\right)$ vanishing along $B_{1}$ determines a degree 5 cyclic covering $f: X \rightarrow Y$, where $X$ is the normalization of $\operatorname{Spec}\left(\oplus_{m=0}^{4} \mathcal{O}_{Y}(-m C)\right)$. This covering is étale outside $B_{1} \cup\left\{p_{1}\right\}$ and totally ramified over $B_{1}$. A local computation over the point $p_{1}$ shows that $X$ is smooth and $f$ is totally ramified over $p_{1}$. We have $-K_{X}=f^{*} C$. Note that $C^{2}=1 / 5$, so $C^{2}$ is ample by Kleiman's criterion. Hence $-K_{X}$ is ample as well and, by projection formula, $K_{X}^{2}=$ 1. To conclude, let $\sigma$ be any generator of the Galois group of $f$. Then $\rho(X)^{\sigma}=\rho(Y)=1$.

\section{§6. Counting the number of automorphisms}

Bertini and Geiser involutions are unique on a given Del Pezzo surface of degree 1 or 2 . Here we consider the same question for the automorphisms described in cases A1-A4 of Theorem A.

\section{Proposition D.}

D1. If $X$ is as in $\mathrm{A} 1$, there are exactly eight distinct automorphisms as in $\mathrm{A} 1$ if $X$ is the Fermat cubic (i.e., if $X$ is defined by $x^{3}+y^{3}+z^{3}+$ $\left.w^{3}=0\right)$, and exactly two otherwise.

D2. If $X$ is as in $\mathrm{A} 2$, it has exactly two distinct automorphisms as in $\mathrm{A} 2$.

D3. If $X$ is as in $\mathrm{A} 3$, it has exactly four distinct automorphisms as in $\mathrm{A} 3$.

D4. If $X$ is as in $\mathrm{A} 4$, it has exactly 24 distinct automorphisms as in $\mathrm{A} 4$.

Proof. Case D1 of the proposition is well known (see for instance [26], page 129). Assume then that $X$ is a smooth sextic surface in $\mathbb{P}(1,1,2,3)$. D2 follows simply by the fact that there is only one linear projection from 
$\mathbb{P}(1,1,2,3)$ onto $\mathbb{P}(1,1,3)$. Concerning case D3, suppose there are two automorphisms $\sigma_{1}, \sigma_{2} \in \operatorname{Aut}(X)$ of order 5 corresponding to two distinct linear projections $\pi_{q_{i}}: \mathbb{P}(1,1,2,3) \rightarrow P_{i} \cong \mathbb{P}(1,2,3)$. Then we can fix coordinates $(x, y, z, w)$ in $\mathbb{P}(1,1,2,3)$ such that $q_{1}=(1,0,0,0), P_{1}=\{x=0\}$, $q_{2}=(a, 1,0,0)$ and $P_{2}=\{b x+c y=0\}$ (we assume that $P_{i} \cap X=R_{i}$, the ramification divisor of $f_{i}$ ). But one can see, by considering how it reflects on the equation of $X$, that this situation is impossible.

Lastly, let $X=\mathrm{Bl}_{\Sigma} \mathrm{P}^{2}$ be the Del Pezzo surface of degree 5. Let $\mathcal{L}$ be the set of lines passing through pairs of points of $\Sigma$. Any splitting of $\mathcal{L}$ into two "triangles" (i.e. into two sets of three lines with no common points) determines a decomposition of the set of $(-1)$-curves of $X$ in two "pentagons". To each distinct splitting of $\mathcal{L}$ as above there correspond four automorphisms of order 5 , and conversely. We conclude counting six possible distinct ways of splitting $\mathcal{L}$.

\section{§7. Proofs of Theorems E and F}

The results proved in the previous sections are finally applied to prove the classification of birational transformations of prime order of $\mathbb{P}^{2}$ (Theorem E) and of their moduli spaces (Theorem F). Part of the the arguments used in the proofs are taken from [4].

\subsection{Proof of Theorem E}

Let $\tau$ be a birational transformation of $\mathbb{P}^{2}$ of prime order $p$. By Theorem 3.5.1, there is a resolution $(X, \sigma)$ of the pair $\left(\mathbb{P}^{2}, \tau\right)$. We can assume that $(X, \sigma)$ is a minimal pair. Note that $K_{X}$ is not nef, thus we can apply to $(X, \sigma)$ the results stated in Theorem A of Chapter 5.

In case 2 of Theorem $\mathrm{A}, X$ is isomorphic to an Hirzebruch surface $\mathbb{F}_{e}$ for some $e \geq 0$. We perform elementary transformations to reduce $(X, \sigma)$ to $\left(\mathbb{F}_{1}, \sigma^{\prime}\right)$, with $\sigma^{\prime} \in \operatorname{Aut}\left(\mathbb{F}_{1}\right)$, in the following way. We can find a fixed point of $X$ not contained in the $(-e)$-curve. Blowing it up and contracting the proper transform of the fiber, we obtain $\mathbb{F}_{e-1}$ if $e \geq 2$, or $\mathbb{F}_{1}$ if $e=0$. Note that $\sigma$ induces there an automorphism. Proceeding in this way, we end up with the desired $\left(\mathbb{F}_{1}, \sigma^{\prime}\right)$. Finally, contracting the $(-1)$-curve of $\mathbb{F}_{1}$, we obtain an automorphism of $\mathbb{P}^{2}$.

We consider now case 4 of Theorem A. By blowing up a fixed point of $X=\mathbb{P}^{1} \times \mathbb{P}^{1}$ and contracting the proper transforms of the two lines through that point, we see that $\sigma$ is birationally equivalent to an automorphism on $\mathbb{P}^{2}$. 
Next, let $(X, \sigma)$ be as in case 3 of Theorem A. The fixed divisor $R \subset X$ is a smooth curve passing to the singular point of each reducible fibers and intersecting each smooth fiber in two distinct points. After contracting one component of each reducible fiber of $X$, we get on a $\mathbb{F}_{e}$, where $\sigma$ induces a birational involution and $R$ is mapped isomorphically. Performing elementary transformations centered at general points of the image of $R$, we eventually get a birational involution $\tau^{\prime}$ on $\mathbb{F}_{1}$. After this birational modification, $R$ is mapped isomorphically to a curve in $\mathbb{F}_{1}$. We will still denote this curve by $R$. Let $E$ and $F$ be respectively the (-1)-curve and a fiber of $\mathbb{F}_{1}$, and write $R=2 E+r F$. Adjunction formula yields $r=g+2$, where $g$ is the genus of $R$, thus $E \cdot R=g$. After further suitable elementary transformations, we can lower the multiplicity of intersection at each point of $R \cap E$ until we get that $R$ and $E$ meet transversally in $g$ distinct points. At this point we blow down $E$, obtaining a birational involution of $\mathbb{P}^{2}$. This involution fixed a curve of degree $d=g+2$ with an ordinary multiple point $q$ of multiplicity $g$ as unique singularity, and lets invariant the lines through $q$. This is a de Jonquières involution of degree $d \geq 2$. To conclude this case, we claim that any de Jonquières involution of degree 2 is conjugate to an automorphism. In analogy with Example 1.3.1, a point $q$ is fixed outside a smooth conic $C$. Let $T_{1}$ and $T_{2}$ be the two lines passing through $p$ and tangent to $C, q_{i}$ be the point of contact of $T_{i}$ with $C$, and $L$ be the line spanned by $q_{1}$ and $q_{2}$. Blowing up $\mathbb{P}^{2}$ at $q_{1}$ and $q_{2}$ and contracting the proper transform of $L$, we get to $\mathbb{P}^{1} \times \mathbb{P}^{1}$, where the de Jonquières involution induces an automorphism $\sigma^{\prime}$. We observe that $\rho\left(\mathbb{P}^{1} \times \mathbb{P}^{1}\right)^{\sigma^{\prime}}=1$, which in particular implies that $\left(\mathbb{P}^{1} \times \mathbb{P}^{1}, \sigma^{\prime}\right)$ is a minimal pair. By Theorem $\mathrm{A}, \sigma^{\prime}$ is the involution which exchanges the two rulings of $\mathbb{P}^{1} \times \mathbb{P}^{1}$, so it is birationally equivalent to an automorphism of $\mathbb{P}^{2}$.

Each case among 5-A4 of Theorem A is clearly birationally equivalent to one of the birational transforms described in Examples 1.3.2-E4 (in the same order). The normalized fixed curve $\operatorname{NFC}(\tau)$ is given by the isomorphism class of the ramification divisor of the cover $X \rightarrow X / \sigma$, and in all cases but A4, this is an irrational curve. This shows that the birational transformations $\tau$ described in Examples 1.3.1-E3 are not conjugate to elements in $\operatorname{Aut}\left(\mathbb{P}^{2}\right)$. Finally, by comparing this invariant together with the order of the transformation, we conclude that all examples determine different conjugacy classes. 


\subsection{Proof of Theorem $\mathbf{F}$}

Let $\left(\mathbb{P}^{2}, \tau\right)$ be one of the pairs listed in Examples 1.3.1-E4 and $(X, \sigma)$ denote its minimal resolution of indeterminacy. We recall that, excluding the de Jonquières involutions, $X$ is a Del Pezzo surface and the resolution of $\left(\mathbb{P}^{2}, \tau\right)$ is given by the blowup of $\mathbb{P}^{2}$ along $\Sigma$.

\section{Proposition 7.2.1. The correspondence NFC is surjective.}

Proof. Let $C$ be an hyperelliptic curve of genus $g \geq 1$ (with abuse of language, among hyperelliptic curve we include here also elliptic curves). Let $g_{2}^{1}$ be a pencil of degree 2 on $C$, and let $p_{1}, \ldots, p_{g} \in C$ be $g$ distinct points such that $p_{i}+p_{j} \notin g_{2}^{1}$ for all pairs $i, j$ (for $g=1$ this condition is empty). The morphism defined by the linear system $\left|g_{2}^{1}+p_{1}+\cdots+p_{g}\right|$ maps $C$ to a plane curve of degree $g+2$ with an ordinary multiple point $q$ of multiplicity $g$ as unique singularity. This shows that NFC is surjective for the case of de Jonquières involutions of degree $d \geq 3$.

For the remaining cases, since $\operatorname{NFC}(\tau)$ is the isomorphism class of the ramification divisor of $f: X \rightarrow X / \sigma$, the claim is clear by Proposition $\mathrm{C}$ except when $\tau$ is as in Example E2. For this case, we have to check that any three-canonical model $C \subset \mathbb{P}^{4}$ of a curve of genus 2 lies on a cone over a rational twisted cubic. Let $W$ be the linear subspace of $\left|3 K_{C}\right|$ spanned by the image of the triple embedding of $\left|K_{C}\right|$ in $\left|3 K_{C}\right|$. Then the linear projection $\pi: \mathbb{P} H^{0}\left(3 K_{C}\right)^{*}=\mathbb{P}^{4} \rightarrow W^{*}=\mathbb{P}^{3}$ maps $C$ two-to-one onto a rational twisted cubic. The required cone is then obtained by taking the closure of $\pi^{-1} \pi(C)$.

\section{Proposition 7.2.2. The correspondence NFC is injective.}

Proof. Let $\tau$ be a de Jonquières involution of degree $d \geq 3$ and $C \in \mathbb{P}^{2}$ its fixed curve. Let $\nu: \mathbb{F}_{1} \rightarrow \mathbb{P}^{2}$ be the blowup at the singular point $p$ of $C$ and $C_{0}=\nu_{*}^{-1} C$. The fibers of $\mathbb{F}_{1}$ cut on $C_{0}$ a $g_{2}^{1}$, and $C_{0} \cap E$ is a set of $g$ distinct points $p_{1}, \ldots, p_{g}$. Clearly $p_{i}+p_{j} \notin g_{2}^{1}$ for all pairs $i, j$ and $\left.\nu\right|_{C_{0}}$ is the morphism defined by $\left|g_{2}^{1}+p_{1}+\cdots+p_{g}\right|$. Then $\tau$ is uniquely determined by $C_{0} \cap E$. Let $p_{g+1}$ be another point of $C$ satisfying $p_{i}+p_{g+1} \notin g_{2}^{1}$ for all $i=1, \ldots, g+1$. After a suitable elementary transformation we can reduce to the case where $C_{0}$ is embedded in $\mathbb{F}_{1}$ in such a way that $C_{0} \cap E=\left\{p_{2}, \ldots, p_{g+1}\right\}$. In this way, by performing further elementary transformations on $\mathbb{F}_{1}$, we can produce a birational link between two any de Jonquières involutions those fixed curves have isomorphic normalization $C_{0}$. 
Let now $\tau$ be one among Examples 1.3.2-E4. In all cases, the isomorphism class of $X$ depends bijectively on the configuration in $\mathbb{P}^{2}$ of the points of $\Sigma$ up to linear action on $\mathbb{P}^{2}$, and the cover $X \rightarrow X / \sigma$ is uniquely determined by its branch divisor up to isomorphism of $X / \sigma$. To conclude, it remains to check that the four different coverings over $\mathbb{P}^{2}$ of the Fermat cubic of $\mathbb{P}^{3}$ determine conjugate cyclic subgroups of $\operatorname{Bir}\left(\mathbb{P}^{2}\right)$. This is clear, since such coverings are transformed one into the other by automorphisms of $X$.

\section{REFERENCES}

[1] M. Alberich-Caraminana, Geometry of the Plane Cremona Maps, Lectures Notes in Math., 1769 (2002), Springer-Verlag, Berlin.

[2] L. Autonne, Reserches sur les groupes d'ordre fini contenus dans le groupe Cremona, J. Math. Pures et Appl. (1885).

[3] W. Barth, C. Peters and A. Van de Ven, Compact Complex Surfaces, Ergebnisse der Mathematik und ihrer Grenzgebiete, 4, Springer-Verlag, Berlin, 1984.

[4] L. Bayle and A. Beauville, Birational involutions of $\mathbb{P}^{2}$, Asian J. Math., 4(1) (2000), $11-18$.

[5] A. Beauville, On Cremona transformations of prime order, Preprint available at ArXiv:math.AG/0402037.

[6] E. Bertini, Ricerche sulle trasformazioni univoche involutorie nel piano, Annali di Mat., 8 (1877), 244-286.

[7] A. Bottari, Sulla razionalità dei piani multipli $\{x, y, \sqrt[n]{F(x, y)}\}$, Ann. Mat. Pura Appl., Serie III, 2 (1899), 277-296.

[8] A. Calabri, On rational and ruled double planes, Ann. Mat. Pura Appl., 181(4) (2002), 365-387.

[9] — On rational cyclic triple planes, Preprint (2001).

[10] G. Castelnuovo and F. Enriques, Sulle condizioni di razionalità dei piani doppi, Rend. del Circ. Mat. di Palermo, 14 (1900), 290-302.

[11] T. de Fernex and L. Ein, Resolution of indeterminacy of pairs, in Algebraic Geometry, a Volume in Memory of Paolo Francia, eds. M. Beltrametti et al. (2002), de Gruiter.

[12] W. Engel, Invariante Divisorensharen dei endlichen Gruppen von Cremonatransformationen, J. Reine Angew. Math., 196 (1956), 59-66.

[13] T. Fujita, Classification theories of polarized varieties, London Mathematical Society Lecture Note Series, 155 (1990), Cambridge Univ. Press, Cambridge.

[14] M. H. Gizatullin, Rational G-surfaces, Izv. Akad. Nauk SSSR Ser. Mat., 44(1) (1980), 110-144, 239.

[15] L. Godeaux, Une représentation des transformations birationnelles du plan et de l'espace, Acad. Roy. Belgique. Cl. Sci. Mém. Coll. in 8. (2), 24(2) (1949), 31.

[16] R. Hartshorne, Algebraic geometry, Graduate Texts in Mathematics, No. 52, Springer-Verlag, New York, 1977, pp. xvi+496. 
[17] T. Hosoh, Automorphism groups of quartic del Pezzo surfaces, J. Algebra, 185(2) (1996), 374-389.

[18] — Automorphism groups of cubic surfaces, J. Algebra, 192(2) (1997), 651-677.

[19] S. Kantor, Theorie der endlichen Gruppen von eindeutigen Transformationen in der Ebene, Mayer \& Müller, Berlin, 1895.

[20] M. Koitabashi, Automorphism groups of generic rational surfaces, J. Algebra, 116(1) (1988), 130-142.

[21] J. Kollár and S. Mori, Birational geometry of algebraic varieties, With the collaboration of C. H. Clemens and A. Corti, Translated from the 1998 Japanese original, Cambridge University Press, Cambridge, 1998, pp. viii+254.

[22] Yu. I. Manin, Rational surfaces over perfect fields. II., Math. USSR Sb., 1 (1967), $141-168$.

[23] Cubic forms: algebra, geometry, arithmetic, Translated from Russian by M. Hazewinkel, North-Holland Mathematical Library, Vol. 4, North-Holland Publishing Co., Amsterdam, 1974, pp. vii+292.

[24] R. Miranda and U. Persson, On extremal rational elliptic surfaces, Math. Z., 193(4) (1986), 537-558.

[25] S. Mori, Threefolds whose canonical bundles are not numerically effective, Ann. of Math. (2), 116(1) (1982), 133-176.

[26] B. Segre, The non-singular cubic surface (1942), Oxford University Press, Oxford.

[27] A. Wiman, Zur theorie der endlichen gruppen von birazionalen transformationen in der ebene, Math. Ann., 4 (1897).

[28] D.-Q. Zhang, Automorphisms of finite order on rational surfaces, J. Algebra, 238(2) (2001), 560-589.

[29] — Automorphisms of finite order on Gorenstein del Pezzo surfaces, Trans. Amer. Math. Soc., 354(12) (2002), 4831-4845.

Department of Mathematics

University of Michigan

East Hall

525 East University Avenue

Ann Arbor, MI 48109-1109

$U S A$

defernex@umich.edu 